\title{
Gravitational-wave generation in hybrid quintessential inflationary models
}

\author{
Paulo M. Sa* \\ Departamento de Física, Faculdade de Ciências e Tecnologia, \\ Universidade do Algarve, Campus de Gambelas, 8005-139 Faro, Portugal \\ Alfredo B. Henriquest \\ Centro Multidisciplinar de Astrofísica - CENTRA and Departamento de Fúsica, \\ Instituto Superior Técnico, UTL, Av. Rovisco Pais, 1049-001 Lisboa, Portugal
}

(Dated: March 18, 2010)

\begin{abstract}
We investigate the generation of gravitational waves in the hybrid quintessential inflationary model. The full gravitational-wave energy spectrum is calculated using the method of continuous Bogoliubov coefficients. The post-inflationary kination period, characteristic of quintessential inflationary models, leaves a clear signature on the spectrum, namely, a peak at high frequencies. The maximum of the peak is firmly located at the $\mathrm{MHz}-\mathrm{GHz}$ region of the spectrum and corresponds to $\Omega_{\mathrm{GW}} \simeq 10^{-12}$. This peak is substantially smaller than the one appearing in the gravitational-wave energy spectrum of the original quintessential inflationary model, therefore avoiding any conflict with the nucleosynthesis constraint on $\Omega_{\mathrm{GW}}$.

PACS numbers: 04.30.Db, 98.70.Vc, 98.80.Cq, 95.36.+x
\end{abstract}

\section{INTRODUCTION}

Gravitational waves of cosmological origin are at present the object of an important research effort, as people realize that they will provide us with a unique telescope to the very early Universe, giving us information not easily available by any other means. Indeed, it would be of the utmost importance to be able to find direct signals coming from these earliest of times, in order to improve our understanding about inflation, preheating and reheating mechanisms, post-inflationary phase transitions, topological defects of grand unified theories, and string theory, among other issues relevant for both cosmology and high-energy physics.

In this work we investigate gravitational-wave generation within a model recently proposed by Bastero-Gil et al. 1], a modification of the original quintessential inflationary model of Peebles and Vilenkin [2]. In the original model, a unified description of inflation and dark energy is achieved with a single scalar field $\phi$ playing both roles of inflaton and quintessence. In such quintessential inflationary models reheating does not proceed in the usual way, through the complete decay of the scalar field into quanta of other fields. In fact, since the potential of the scalar field has no minimum, inflation is followed not by coherent oscillations of the scalar field, but rather by a kination period [3, 4], during which the evolution is dominated by the kinetic energy of the scalar field $\phi$, which behaves approximately as stiff matter. Reheating proceeds then by gravitational particle production taking place at the transition from the inflationary to the kination period [5]. This mechanism is, however, quite ineffi-

\footnotetext{
*Electronic address: pmsa@ualg.pt

${ }^{\dagger}$ Electronic address: alfredo.henriques@ist.utl.pt
}

cient and may lead to cosmological problems associated with large isocurvature fluctuations and overproduction of gravitinos and moduli fields [6]. In order to recover the usual reheating mechanism, Bastero-Gil et al. [1] introduce another scalar field $\chi$, coupled to the original inflaton/quintessence field $\phi$, with a hybrid-like potential [7. In such a hybrid quintessential inflationary model, during the kination period driven by the kinetic energy of the field $\phi$, the field $\chi$ oscillates around the minimum of the hybrid-like potential, completely decaying into relativistic particles, thus reheating the Universe. Since this reheating mechanism is more efficient than gravitational particle production, the radiation-dominated epoch of expansion of the Universe begins earlier and, therefore, the kination period is shorter in this hybrid model than in the original one.

It is known that a phase of evolution in the early Universe with an equation of state stiffer than radiation leads to a sharp increase of the gravitational-wave spectral energy density parameter $\Omega_{\mathrm{GW}}$ in the high-frequency region of the spectrum [8 10]. This peak in the gravitationalwave spectrum is unavoidable in quintessential inflationary models, in which inflation is followed by a period of kination $11-14$. In the original quintessential inflationary model of Peebles and Vilenkin [2], the height of the peak depends on the number of scalar degrees of freedom, $N_{s}$, whose decay into fermions triggers the onset of a gravitational reheating of the Universe. For the minimum allowed value of $N_{s}$, of the order of $10^{2}$, the gravitational-wave spectral energy density parameter $\Omega_{\mathrm{GW}}$ could be as high as $10^{-6}$ [1]], several orders of magnitude higher than in standard inflation. Clearly, in the hybrid quintessential inflationary model, in which the kination period is shorter than in the original model, one expects the high-frequency peak of the spectrum to be smaller.

Our main aim in this paper will be to calculate, within 
the hybrid quintessential inflationary model, the presentday spectrum of the cosmological gravitational waves generated during the evolution of the Universe and to show that its careful analysis will allow us to extract much information about the properties of the potential driving both the primordial inflationary stage and the present-day accelerated expansion. To take into account the expansion of the Universe during the transitions between the different stages of its evolution, characterized by different equations of state, we use throughout the method first developed by Parker, in his seminal paper of 1969 [15]. This method, applied by Parker to particle production in an expanding Universe and then extended to the case of gravitons [16 18], is based on the time evolution of the Bogoliubov coefficients, which obey appropriate differential equations. The numerical integration of these equations will immediately allow us to construct the full spectrum of the gravitational waves (see Refs. [19 22] for applications of this method to several cosmological models).

Our paper is organized as follows. In the next section we describe the hybrid quintessential inflationary model of Bastero-Gil et al. [1] and write the equations of motion for the different stages of the expansion. We also describe the simple phenomenological mechanism responsible for the reheating of the Universe, acting during the kination period that follows inflation. In section III, we present the aforementioned method of the continuous Bogoliubov coefficients to calculate the spectrum of the gravitational waves, integrate the corresponding equations and compute the gravitational-wave spectral energy density parameter for frequencies ranging from about $10^{-17} \mathrm{rad} / \mathrm{s}$ to about $10^{10} \mathrm{rad} / \mathrm{s}$. The influence on the spectrum of the various parameters defining the hybrid quintessential inflationary potential and the resulting constraints are then carefully analyzed in the different frequency regions. In section IV we summarize our main conclusions.

\section{HYBRID QUINTESSENTIAL INFLATION}

\section{A. The hybrid potential}

As shown by Peebles and Vilenkin [2], the unification of inflation and dark energy within a single framework, which they termed quintessential inflation, can be achieved with a single scalar field with potential given by

$$
V(\phi)= \begin{cases}\lambda_{\phi}\left(\phi^{4}+M^{4}\right), & \text { for } \quad \phi<0, \\ \lambda_{\phi} M^{8}\left(\phi^{4}+M^{4}\right)^{-1}, & \text { for } \phi \geqslant 0,\end{cases}
$$

where $\lambda_{\phi}$ and $M$ are constants. The value of $\lambda_{\phi}$ is constrained by recent measurements of the cosmic microwave background and large-scale structure to be of the order of $10^{-13}$ (see Ref. [23] for a derivation of such constraints for different models of inflation). On the other hand, agreement with the measured value of today's dark-energy density [24] requires $M$ to be of the order of $10^{-14} m_{\mathrm{p}}$.

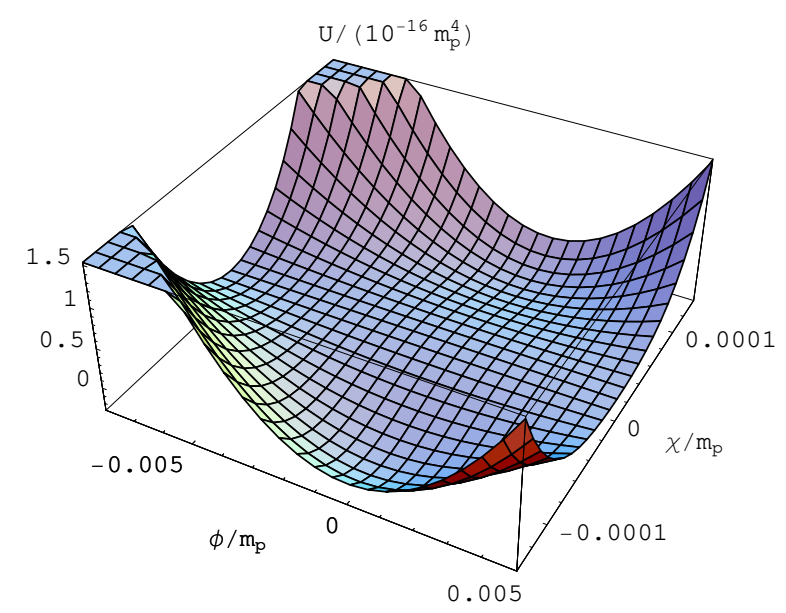

FIG. 1: Potential $U(\phi, \chi)$ for $m=0.004 m_{\mathrm{p}}, \lambda_{\phi}=10^{-13}$, $M=1.7 \times 10^{-14} m_{\mathrm{p}}, \lambda_{\chi}=1$ and $g=0.025$.

Recently, Bastero-Gil et al. [1] proposed a modification of the original quintessential inflationary model, in which the reheating of the Universe is driven not by gravitational particle production but rather by the simpler mechanism of the decay of an oscillating massive field. This is achieved by the introduction of an extra scalar field $\chi$ with a hybrid-like potential given by

$$
U(\phi, \chi)=V(\phi)+\frac{1}{2} g^{2} \chi^{2}\left(\phi^{2}-m^{2}\right)+\frac{1}{4} \lambda_{\chi} \chi^{4},
$$

where $V(\phi)$ is given by Eq. (11) and $g, m$, and $\lambda_{\chi}$ are some parameters to be specified. Note that for $|\phi| \geqslant$ $m$ the potential (2) has just one minimum at $\chi=0$, while for $|\phi|<m$ it has two minima located at $\chi=$ $\pm \sqrt{g^{2}\left(m^{2}-\phi^{2}\right) / \lambda_{\chi}}$ (see Fig. 1).

In our analysis of this hybrid quintessential inflationary model we will divide the evolution of the Universe into four stages.

In the first stage of evolution, $\phi \leqslant-m$ and $\chi$ is assumed to be at rest at the minimum of the potential $(\chi=0)$. Initial conditions are such that the potential energy of the scalar field $\phi$ dominates the evolution of the Universe, yielding a period of chaotic inflation. By the end of this stage of evolution, the kinetic term in the energy density of $\phi$ becomes dominant, giving rise to a period of kination, during which the scalar field behaves as stiff matter with equation of state $p=w \rho, w=1$.

During the second stage of evolution, which takes place for $|\phi|<m$, the origin of the potential becomes unstable for the scalar field $\chi$. As a result, this field rolls down toward one of the new minima of the potential, located at $\chi= \pm \sqrt{g^{2}\left(m^{2}-\phi^{2}\right) / \lambda_{\chi}}$, and starts to oscillate around it. Of course, this behavior of the scalar field $\chi$ is expected only if the duration of the second stage of evolution is much greater than the characteristic response time of the field to changes in the potential. As shown in Ref. [1], this requirement can be formulated as 
a constraint on the values of the parameters $g$ and $m$, namely,

$$
g\left(\frac{m}{m_{\mathrm{p}}}\right)^{2} \gtrsim 0.2 \lambda_{\phi}^{1 / 2}
$$

We also demand that the motion of the scalar field $\phi$ is not influenced significantly by $\chi$, i.e., we impose the condition that the energy density of $\chi$ is much smaller than the kinetic term in the energy density of $\phi$ during the second stage of evolution. This condition can be also translated into a constraint on the values of the parameters appearing in the potential (2), namely [1],

$$
\frac{g^{4}}{\lambda_{\chi}}\left(\frac{m}{m_{\mathrm{p}}}\right)^{4} \lesssim 0.001 \lambda_{\phi}
$$

Note that this last condition implies that kination extends throughout the second stage of evolution.

The third stage of evolution takes place for $\phi \geqslant m$. For such values of the scalar field $\phi$, the potential (2) has again only one minimum in the $\chi$ direction, located at the origin. The scalar field $\chi$ oscillates around this stable minimum, transferring its kinetic energy, acquired during the second stage of evolution, to a radiation fluid, thus reheating the Universe. This decay of the oscillating scalar field $\chi$ into radiation is achieved by the introduction of a phenomenological dissipative coupling, between the scalar field $\chi$ and the radiation fluid, proportional to the mass of $\chi[1,25]$,

$$
\Gamma_{\chi}=\mu m_{\chi}=\mu g \sqrt{\phi^{2}-m^{2}},
$$

$\mu$ being the proportionality constant. The abovementioned condition that the motion of the scalar field $\phi$ is not influenced significantly by $\chi$ also imposes a constraint on the values of $\mu$, namely [1],

$$
\mu \gg 0.2 \lambda_{\phi}^{1 / 6}\left(\frac{m_{\mathrm{p}}}{m}\right)^{2 / 3} \frac{g^{5 / 3}}{\lambda_{\chi}} .
$$

Note that this constraint, as well as constraints (3) and (41), was derived assuming that $m \ll m_{\mathrm{p}}{ }^{1}$. During the third stage of evolution, the energy density of $\chi$ decays away rapidly. Gradually, as the energy density of radiation increases, kination gives place to a radiationdominated Universe.

The fourth stage of evolution extends from the beginning of the radiation-dominated era to the present epoch.

\footnotetext{
1 The constraints (3), (4), and (6) differ from the ones derived in Ref. 1] by some numerical factors. This is due to the fact that Bastero-Gil et al. estimated the scale-factor growth between the end of the inflationary period and the beginning of the second stage of evolution to be of the order of 8 , while our numerical simulations show this growth to be half of this value. We take into account this factor 2 , since the constraints involve third and sixth powers of the scale-factor growth.
}

The scalar field $\chi$ is assumed to have decayed away completely in the previous stage of evolution. A matter component (dark and usual, baryonic, matter) is introduced into the equations of motion, giving rise to a intermediate matter-dominated period in the evolution of the Universe. The scalar field $\phi$, which in the first stage of evolution played the role of inflaton, stays now practically constant and, at late times, begins to dominate the evolution of the Universe, giving rise to the present epoch of accelerated expansion. Therefore, the scalar field $\phi$ plays, within this model, both roles of inflaton and quintessence.

\section{B. The evolution of the Universe}

Let us now present the equations of motion for the different stages of evolution.

For the first and second stages of evolution, the equations of motion are

$$
\begin{gathered}
\frac{\ddot{a}}{a}=-\frac{8 \pi}{3 m_{\mathrm{p}}^{2}}\left(\dot{\phi}^{2}+\dot{\chi}^{2}-U\right), \\
\ddot{\phi}+3 \frac{\dot{a}}{a} \dot{\phi}+\frac{\partial U}{\partial \phi}=0, \\
\ddot{\chi}+3 \frac{\dot{a}}{a} \dot{\chi}+\frac{\partial U}{\partial \chi}=0, \\
\left(\frac{\dot{a}}{a}\right)^{2}=\frac{8 \pi}{3 m_{\mathrm{p}}^{2}}\left(\frac{\dot{\phi}^{2}}{2}+\frac{\dot{\chi}^{2}}{2}+U\right),
\end{gathered}
$$

where we have assumed a flat Friedmann-RobertsonWalker metric, $a$ is the scale factor, $m_{\mathrm{p}}$ is the Planck mass $^{2}$, and a dot denotes a derivative with respect to the cosmic time $t$.

A Runge-Kutta method is used to solve the system of differential equations (7)-(91), while Eq. (10) is used as a constraint equation to check the accuracy of the numerical solution.

As initial condition for the scalar field $\phi$ we choose $\phi_{i 1}=-5 m_{\mathrm{p}}$, which guarantees enough inflation. The scalar field $\chi$ is located near the origin; for numerical convenience, we assume $\chi_{i 1}=\dot{\chi}_{i 1}=0$. The initial values of the other variables are chosen to be $a_{i 1}=1$ and $\dot{\phi}_{i 1}=\sqrt{2 U\left(\phi_{i 1}, \chi_{i 1}\right)}$, while $\dot{a}_{i 1}$ is fixed by the Friedmann equation (10). As mentioned above, the parameters $\lambda_{\phi}$ and $M$ of the potential $V(\phi)$ are constrained by observations to be of the order of $10^{-13}$ and $10^{-14} m_{\mathrm{p}}$, respectively. The first stage of evolution ends when $\phi=-m$; for the parameter $m$ we choose $m \lesssim 10^{-2} m_{\mathrm{p}}$.

For such values of the initial conditions and of the parameters $\lambda_{\phi}, M$, and $m$, the first stage of evolution

\footnotetext{
${ }^{2}$ In the first, second, and third stages of evolution we use the natural system of units, with $\hbar=c=1$ and $m_{\mathrm{p}}=G^{-1 / 2}=1.22 \times$ $10^{19} \mathrm{GeV}$, while in the fourth stage we use the International Systems of Units.
} 


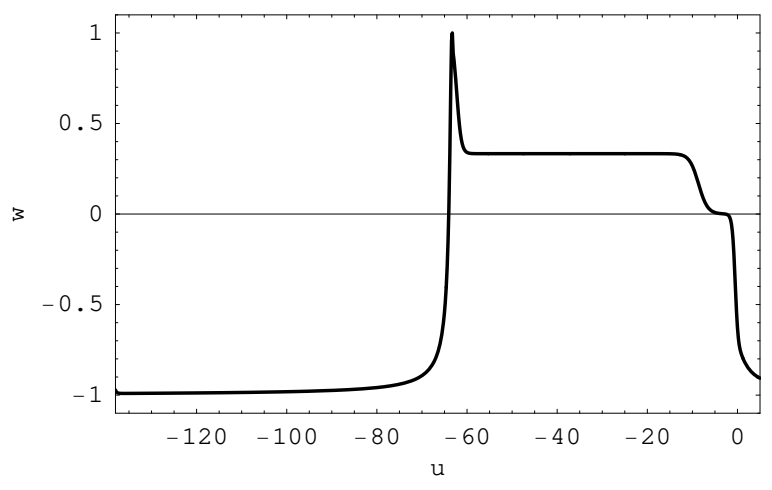

FIG. 2: Time evolution of the equation-of-state parameter $w=p / \rho$, from the beginning of the inflationary period until the present epoch, for $\lambda_{\phi}=10^{-13}, M=1.7 \times 10^{-14} m_{\mathrm{p}}$, $g=0.025, m=0.004 m_{\mathrm{p}}, \lambda_{\chi}=1$, and $\mu=0.1$. In this figure, instead of the cosmic time $t$, we use the variable $u=$ $-\ln (1+z)$, where $z$ is the redshift. The first stage of evolution corresponds to $-139 \lesssim u \lesssim-63$, the second stage to $u \approx-63$, the third stage to $-63 \lesssim u \lesssim-60$, and the fourth stage to $-60 \lesssim u \leqslant 0$. The epochs of inflation, kination, radiation domination, matter domination, and dark-energy domination can be clearly identified in this figure.

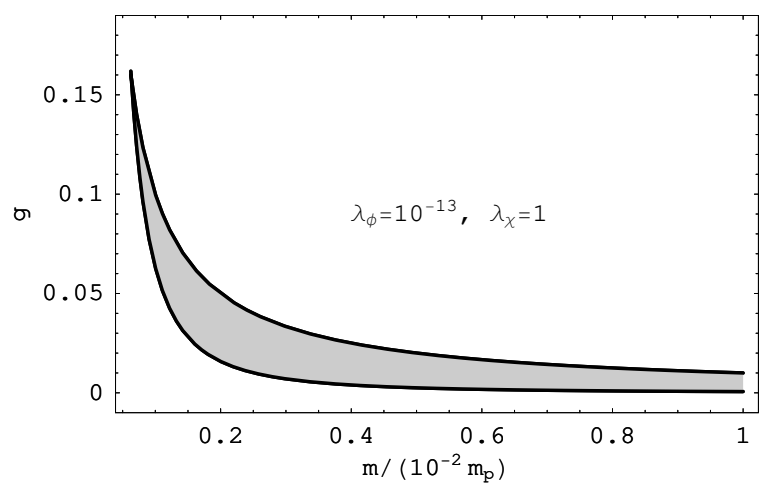

FIG. 3: Allowed values of the parameters $m$ and $g$ (shaded region), for $\lambda_{\phi}=10^{-13}$ and $\lambda_{\chi}=1$.

lasts for about $10^{7} t_{\mathrm{p}}$ and the scale factor grows about 32 orders of magnitude. The equation-of-state parameter $w=p / \rho$ changes gradually from $w=-1$ (inflation) to $w=1$ (kination), as shown in Fig. 2.

At the beginning of the second stage of evolution, the origin becomes unstable for the scalar field $\chi$. We perturb slightly this field, making it roll towards the temporary minimum located at $\chi_{\min }(\phi)=+\sqrt{g^{2}\left(m^{2}-\phi^{2}\right) / \lambda_{\chi}}$. More exactly, we choose $\chi_{i 2}=0.01 \chi_{\min }(0)$ and $\dot{\chi}_{i 2}=0$. The parameters $g, m$, and $\lambda_{\chi}$ should satisfy the constraints (3) and (4). We also demand that $g \lesssim 1, \lambda_{\chi} \lesssim 1$, and $m \lesssim 10^{-2} m_{\mathrm{p}}$. If, for instance, we choose $\lambda_{\chi} \approx 1$, then the values of $g$ and $m$ are restricted to the shaded region shown in Fig. 3 For such values of the parameters the second stage of evolution lasts typically for about $10^{6} t_{\mathrm{p}}$ (see Fig. (4).

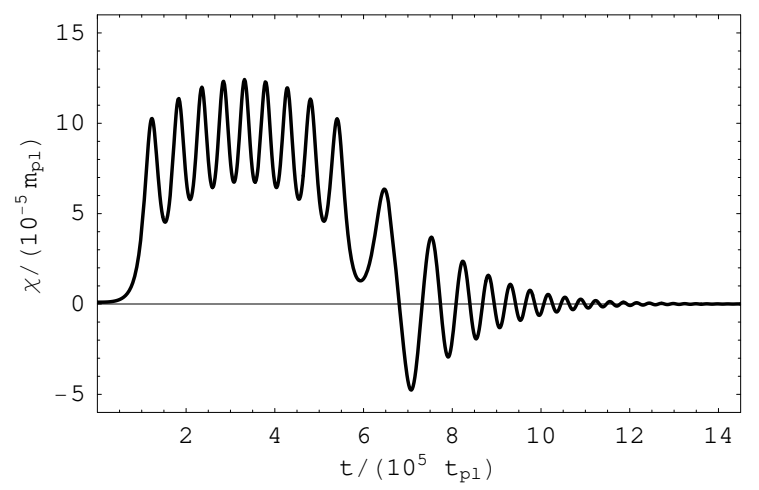

FIG. 4: Time evolution of the scalar field $\chi$ during the second and third stages of evolution, for the same values of the parameters as in Fig. 2 The second stage of evolution lasts about $7 \times 10^{5} t_{\mathrm{p}}$; the third stage of evolution, which lasts about $5 \times 10^{10} t_{\mathrm{p}}$, is only partially shown is this figure.

During the third stage, the evolution of the Universe is described by the set of differential equations

$$
\begin{gathered}
\frac{\ddot{a}}{a}=-\frac{8 \pi}{3 m_{\mathrm{p}}^{2}}\left(\dot{\phi}^{2}+\dot{\chi}^{2}-U+\rho_{r}\right), \\
\ddot{\phi}+3 \frac{\dot{a}}{a} \dot{\phi}+\frac{\partial U}{\partial \phi}=0 \\
\ddot{\chi}+3 \frac{\dot{a}}{a} \dot{\chi}+\frac{\partial U}{\partial \chi}=-\Gamma_{\chi} \dot{\chi} \\
\dot{\rho}_{r}+4 \frac{\dot{a}}{a} \rho_{r}=\Gamma_{\chi} \dot{\chi}^{2} \\
\left(\frac{\dot{a}}{a}\right)^{2}=\frac{8 \pi}{3 m_{\mathrm{p}}^{2}}\left(\frac{\dot{\phi}^{2}}{2}+\frac{\dot{\chi}^{2}}{2}+U+\rho_{r}\right),
\end{gathered}
$$

where $\rho_{r}$ is the energy density of radiation and $\Gamma_{\chi}$ is the dissipative coefficient given by Eq. (5).

Again, we use a Runge-Kutta method to solve the system of differential equations (11)-(14). Equation (15) is used to check the accuracy of the numerical solution. Since any pre-existing radiation fluid would have been diluted during inflation, we choose the energy density of radiation at the beginning of the third stage of evolution to be zero, $\rho_{r, i 3}=0$. The dissipation parameter $\mu$ should satisfy the constraint (6). For $\lambda_{\chi}=1, m=0.004 m_{\mathrm{p}}$, and $g=0.025$ this implies $\mu \gg 10^{-4}$.

As already mentioned above, during the third stage of evolution, the scalar field $\chi$ oscillates around the minimum of the potential located at $\chi=0$ (see Fig. (4), transferring its energy into the radiation fluid (see Fig. (5)), thus reheating the Universe. The reheating temperature depends on the values of the parameters of the model [1]. For the above-mentioned values of the parameters, our numerical simulations show that the reheating temperature is of the order of $10^{14} \mathrm{GeV}$.

For low values of the parameter $\mu$, the scalar field $\chi$ oscillates for a long period of time before its energy is completely transferred to the radiation fluid. In such cases, 


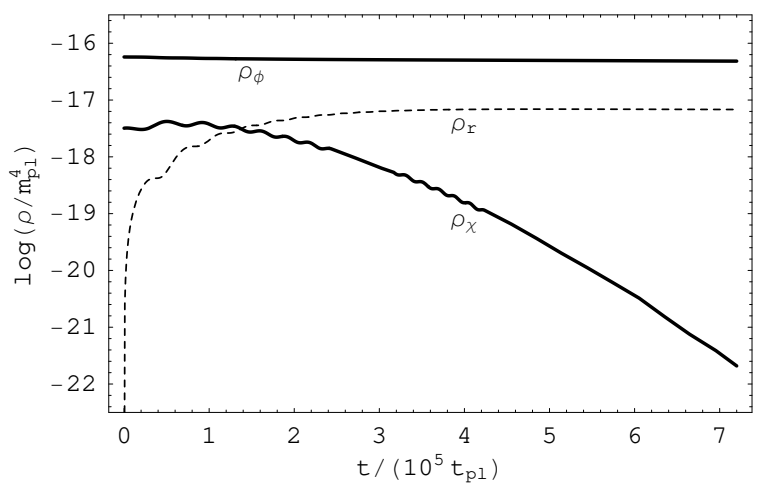

FIG. 5: Time evolution of the energy densities of radiation, $\phi$, and $\chi$ at the beginning of the third stage of evolution (which lasts about $5 \times 10^{10} t_{\mathrm{p}}$ ), for the same values of the parameters as in Fig. 2 Energy is transferred from the scalar field $\chi$ to radiation, while the evolution of the Universe is dominated by the scalar field $\phi$. Later in this stage, the energy density of radiation becomes dominant; kination gives place to a radiation-dominated Universe.

because of the difficulty to accurately follow many oscillations of $\chi$ with our numerical code, a different strategy has to be used. We start by numerically solving Eqs. (11)-(14). After a short period of integration, the terms $g^{2} m^{2} \chi^{2} / 2$ and $\lambda_{\chi} \chi^{4} / 4$ in the potential $U(\phi, \chi)$ become much smaller than $g^{2} \phi^{2} \chi^{2} / 2$, implying that the energy density of the scalar field $\chi$ is given, within a good approximation, by

$$
\rho_{\chi} \approx \frac{\dot{\chi}^{2}}{2}+\frac{1}{2} g^{2} \phi^{2} \chi^{2}
$$

Taking into account that $\phi$ remains practically constant during an oscillation of $\chi$, the oscillations of the latter field can be considered to be of the simple harmonic type, for which $\rho_{\chi}=\left\langle\dot{\chi}^{2}\right\rangle=g^{2} \phi^{2}\left\langle\chi^{2}\right\rangle$, where $\langle\ldots\rangle$ denotes the average over one oscillation. Using this approximation, Eqs. (11)-(15) can be re-written as

$$
\begin{gathered}
\frac{\ddot{a}}{a}=-\frac{8 \pi}{3 m_{\mathrm{p}}^{2}}\left(\dot{\phi}^{2}-V+\frac{1}{2} \rho_{\chi}+\rho_{r}\right), \\
\ddot{\phi}+3 \frac{\dot{a}}{a} \dot{\phi}+\frac{\partial V}{\partial \phi}=-\frac{\rho_{\chi}}{\phi}, \\
\dot{\rho}_{\chi}+3 \frac{\dot{a}}{a} \rho_{\chi}=-\Gamma_{\chi} \rho_{\chi}+\frac{\dot{\phi}}{\phi} \rho_{\chi}, \\
\dot{\rho}_{r}+4 \frac{\dot{a}}{a} \rho_{r}=\Gamma_{\chi} \rho_{\chi}, \\
\left(\frac{\dot{a}}{a}\right)^{2}=\frac{8 \pi}{3 m_{\mathrm{p}}^{2}}\left(\frac{\dot{\phi}^{2}}{2}+V+\rho_{\chi}+\rho_{r}\right) .
\end{gathered}
$$

We then solve these equations, instead of Eqs. (11)-(14), until the end of the third stage of evolution.

Let us now turn to the fourth stage of evolution. The scalar field $\chi$ has already decayed away completely in the previous stage of evolution. Therefore, Eq. (14), or Eq. (20), can be integrated exactly, yielding $\rho_{r}=$ $\rho_{r, 0}\left(a_{0} / a\right)^{4}$, where $\rho_{r, 0}=4.13 \times 10^{-14} \mathrm{~J} / \mathrm{m}^{3}$ and $a_{0}$ are, respectively, today's values of the energy density of radiation and of the scale factor. In this stage of evolution a pressureless matter component is introduced, which accounts for the usual (baryonic) matter and also for dark matter, with energy density $\rho_{m}=\rho_{m, 0}\left(a_{0} / a\right)^{3}$, where $\rho_{m, 0}=2.34 \times 10^{-10} \mathrm{~J} / \mathrm{m}^{3}$ is today's value of the energy density of matter. Taking all this into account, the equations of motion for the fourth stage of evolution are given by

$$
\begin{gathered}
\frac{\ddot{a}}{a}=-\frac{8 \pi G}{3 c^{2}}\left[\dot{\phi}^{2}-V+\rho_{r, 0}\left(\frac{a_{0}}{a}\right)^{4}+\frac{1}{2} \rho_{m, 0}\left(\frac{a_{0}}{a}\right)^{3}\right], \\
\ddot{\phi}+3 \frac{\dot{a}}{a} \dot{\phi}+\frac{\partial V}{\partial \phi}=0 \\
\left(\frac{\dot{a}}{a}\right)^{2}=\frac{8 \pi G}{3 c^{2}}\left[\frac{\dot{\phi}^{2}}{2}+V+\rho_{r, 0}\left(\frac{a_{0}}{a}\right)^{4}+\rho_{m, 0}\left(\frac{a_{0}}{a}\right)^{3}\right],
\end{gathered}
$$

where $G$ is the gravitational constant and $c$ is the speed of light.

This set of differential equations describes a stage of evolution in which the Universe is dominated, consecutively, by radiation, matter and the inflaton/quintessence field $\phi$. As already mentioned above, in order to obtain agreement with observations [24], the parameter $M$ of the potential $V(\phi)$ has to be chosen such that

$$
\left[\frac{\dot{\phi}^{2}}{2}+V(\phi)\right]_{t=t_{0}}=\rho_{d e, 0},
$$

where $\rho_{d e, 0}=6.20 \times 10^{-10} \mathrm{~J} / \mathrm{m}^{3}$ is today's value of the energy density of dark energy. Within the present model, the value of $M$ is typically of the order of $10^{-14} m_{\mathrm{p}}$.

Note that the values of $\rho_{d e, 0}, \rho_{m, 0}$, and $\rho_{r, 0}$ used in this article imply a value of the Hubble constant of $H_{0}=$ $71 \mathrm{~km} \mathrm{~s}^{-1} \mathrm{Mpc}^{-1}$.

\section{GRAVITATIONAL WAVES}

Gravitational waves are generated in an expanding Universe, giving rise to a spectrum extending over a wide range of frequencies, from about $10^{-17} \mathrm{rad} / \mathrm{s}$ to about $10^{10} \mathrm{rad} / \mathrm{s}[2632]$.

In this article, we calculate this spectrum for the hybrid quintessential inflationary model using the method of the continuous Bogoliubov coefficients. This method can be summarized as follows (for details, see Refs. [1622]). The number of gravitons at a certain moment of the evolution of the Universe is given by the squared Bogoliubov coefficient, $\beta^{2}=(X-Y)^{2} / 4$, where the functions $X(t)$ and $Y(t)$ are solutions of the system of differential equations

$$
\dot{X}=-i \omega_{0} \frac{a_{0}}{a} Y
$$




$$
\dot{Y}=-\frac{i}{\omega_{0}} \frac{a}{a_{0}}\left[\omega_{0}^{2}\left(\frac{a_{0}}{a}\right)^{2}-\frac{\ddot{a}}{a}-\left(\frac{\dot{a}}{a}\right)^{2}\right] X ;
$$

$a_{0}$ and $\omega_{0}$ are today's values of the scale factor and the gravitational-wave angular frequency, respectively. The above system of equations is integrated with initial conditions $X\left(t_{i 1}\right)=Y\left(t_{i 1}\right)=1$, corresponding to the absence of gravitons at the beginning of the first stage of evolution. The scale factor $a(t)$ and its first and second derivatives $\dot{a}(t)$ and $\ddot{a}(t)$ are determined from the evolutionary equations presented in the previous section. Knowing $\beta^{2}(t)$, we can compute the gravitational-wave spectral energy density parameter, $\Omega_{\mathrm{GW}}$, which is defined as

$$
\Omega_{\mathrm{GW}}=\frac{8 \hbar G}{3 \pi c^{5} H^{2}} \omega^{4} \beta^{2},
$$

where $H(t)$ is the Hubble parameter. Evaluating all the quantities in the above expression at the present time, for angular frequencies ranging from $1.4 \times 10^{-17} \mathrm{rad} / \mathrm{s}$ (corresponding to a wavelength equal, today, to the Hubble distance) to about $10^{10} \mathrm{rad} / \mathrm{s}$ (corresponding to a wavelength equal to the Hubble distance at the end of the inflationary period), yields the gravitational-wave energy spectrum. It is worth emphasizing that this spectrum is fully determined by the evolution of the scale factor from the beginning of the inflationary period until the present time. A change in the behavior of the scale factor, due to a change of the equation of state of the Universe (see Fig. 2), leads to a modification of the slope of the gravitational-wave spectrum at a certain frequency.

Let us now compute the gravitational-wave spectrum of the hybrid quintessential inflationary model using the formalism of the continuous Bogoliubov coefficients. First, we have to specify the values of $\lambda_{\phi}$ and $M$, satisfying the observational constraints on inflation and dark energy, and the values of $g, m, \lambda_{\chi}$, and $\mu$, satisfying the constraints (3)-(6). In Fig. 6 we show the full gravitational-wave spectrum for the hybrid quintessential inflationary model for two different values of the parameter $g$, corresponding to the minimum and maximum values allowed by the constraints (3) and (4) for fixed values of $\lambda_{\phi}, m$, and $\lambda_{\chi}$.

As we can see from Fig. [6 the spectrum is naturally divided in three regions.

In the low-frequency region, $\omega_{0}$ ranges from $1.4 \times$ $10^{-17} \mathrm{rad} / \mathrm{s}$ to about $2 \pi H\left(t_{m}\right) a\left(t_{m}\right) / a_{0} \approx 10^{-15} \mathrm{rad} / \mathrm{s}$, where the upper limit corresponds to today's value of the angular frequency of a gravitational wave which had a wavelength equal to the Hubble distance at the time $t_{m}$ when the energy density of radiation became equal to the energy density of matter. In this region of the spectrum, the gravitational-wave spectral energy density parameter $\Omega_{\mathrm{GW}}$ rises rapidly as $\omega_{0}$ decreases, due to an extra graviton production during the transition between the radiation- and the matter-dominated eras and the subsequent matter- and dark energy-dominated eras. For $\omega_{0}=1.4 \times 10^{-17} \mathrm{rad} / \mathrm{s}$ the spectrum satisfies a constraint

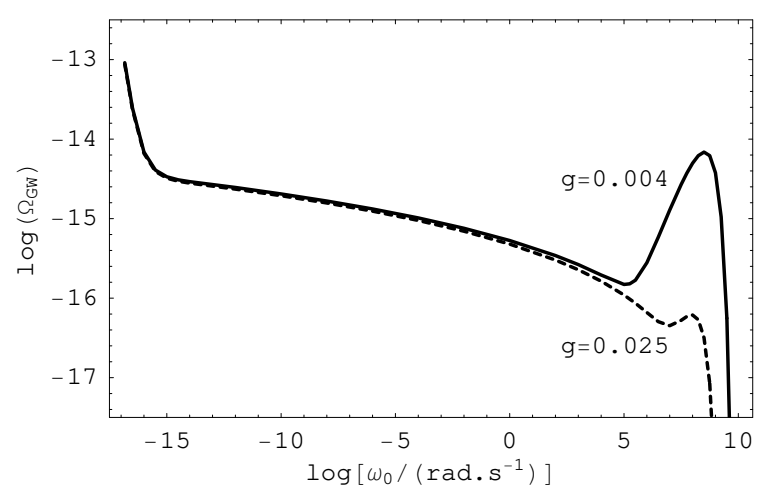

FIG. 6: Gravitational-wave spectrum for $\lambda_{\phi}=10^{-13}, m=$ $0.004 m_{\mathrm{p}}, \lambda_{\chi}=1$, and $\mu=0.1$. The two curves correspond to the minimum and maximum values of $g$ allowed by the constraints (3) and (4), namely, $g=0.004$ and $g=0.025$. In both cases $M$ is of the order of $10^{-14} m_{\mathrm{p}}$.

derived from measurements of the cosmic microwave background radiation, namely, $\Omega_{\mathrm{GW}}<1.4 \times 10^{-10}[\underline{32}$.

In the intermediate-frequency region, the angular frequency ranges from about $10^{-15} \mathrm{rad} / \mathrm{s}$ to $2 \pi H\left(t_{r}\right) a\left(t_{r}\right) / a_{0}$, where $t_{r}$ corresponds to the transition between the kination and radiation-dominated eras, occurring at the end of the third stage of evolution. Depending on the duration of the kination era, the intermediate-frequency region extends up to values of about $\left(10^{3}-10^{7}\right) \mathrm{rad} / \mathrm{s}$. Note that the spectrum has, in this region, a nonconstant slope, due to the fact that inflation is quasi-exponential ${ }^{3}[22$, 33]. Several bounds on the gravitational-wave spectral energy density parameter should be satisfied in this region of the spectrum, namely, from timing observations of millisecond pulsars, $\Omega_{\mathrm{GW}}<4 \times 10^{-8}$ for $\omega_{0}=2.5 \times 10^{-8} \mathrm{rad} / \mathrm{s}$ [34], from Doppler tracking of the Cassini spacecraft, $\Omega_{\mathrm{GW}}<0.028$ for $\omega_{0}=7.5 \times 10^{-6} \mathrm{rad} / \mathrm{s}$ 35], and from the Laser Interferometer Gravitational Wave Observatory (LIGO), $\Omega_{\mathrm{GW}}<6.9 \times 10^{-6}$ for $\omega_{0} \approx\left(10^{2}-10^{3}\right) \mathrm{rad} / \mathrm{s}$ 36.

In the high-frequency region of the spectrum, ranging from $\left(10^{3}-10^{7}\right) \mathrm{rad} / \mathrm{s}$ to about $10^{10} \mathrm{rad} / \mathrm{s}$, one observes a sharp rise of the gravitational-wave spectral energy density parameter $\Omega_{\mathrm{GW}}$. This rather interesting feature of the spectrum is due to the existence of a kination period (with equation of state $p=w \rho, w=1$ ) between the end of inflation and the beginning of the radiationdominated era, whose duration determines the height of the peak [10].

Our numerical simulations show that the duration of the kination period increases as the value of the parameter $g$ decreases (see Fig. 7). This can be easily under-

\footnotetext{
${ }^{3}$ In exponential inflation (cosmological constant) the slope of the spectrum in the intermediate-frequency region is zero [27], while in power-law inflation the slope is constant and negative [30].
} 


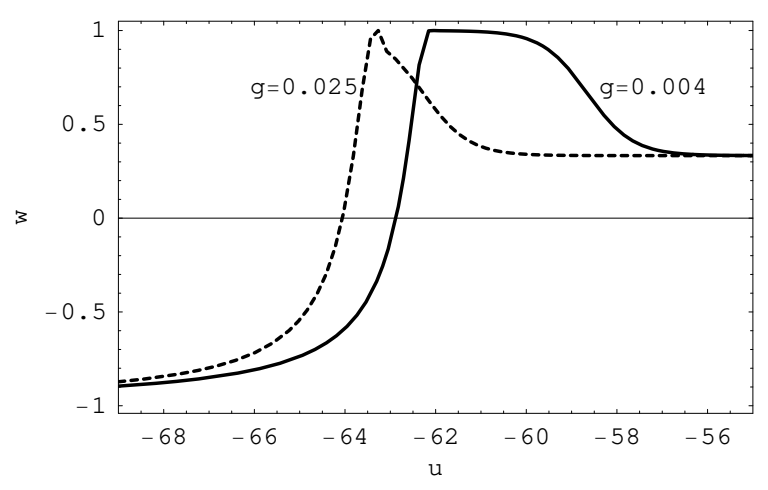

FIG. 7: Time evolution of the equation-of-state parameter $w=p / \rho$ for $g=0.004$ and $g=0.025$. The duration of the kination period in the former case is much longer, implying a higher peak in the gravitational-wave spectrum. In this figure the values of the parameters are the same as in Fig. 6 and $u=-\ln (1+z)$, where $z$ is the redshift.

stood as follows. If one decreases $g$ (for fixed values of $m$ and $\lambda_{\chi}$ ), the value of $\chi$ at the minimum of the potential also decreases (recall that $\chi_{\min }= \pm \sqrt{g^{2}\left(m^{2}-\phi^{2}\right) / \lambda_{\chi}}$ ), meaning that less energy is acquired by this field as it oscillates around the minimum during the second stage of evolution. As a consequence, less energy is available to be transferred, during the third stage of evolution, from the scalar field $\chi$ to the radiation fluid, leading to a lower reheating temperature. This, in turn, implies that after the complete decay of $\chi$ a longer period of time is required for the energy density of radiation to become greater than the kinetic energy of the scalar field $\phi$, i.e., the beginning of the radiation-dominated era is delayed and, consequently, the kination period becomes longer. In short, we could say that reheating becomes less efficient as $g$ decreases.

As we have seen in Sect. III $g$ is not a free parameter, its value is bounded from above by the condition that the motion of $\phi$ is not affected by $\chi$ [see Eq. (4)] and from below by the condition that the scalar field $\chi$ responds quickly enough to changes in the potential $U(\phi, \chi)$, rolling down toward one of the new minima and oscillating around it [see Eq. (3)]. Therefore, within the hybrid quintessential inflationary model, the duration of the kination period and, consequently, the height of the peak in the gravitational-wave spectrum, cannot be freely adjusted, they are limited by the allowed values of the parameter $g$.

What in the previous two paragraphs was said relatively to the dependence of the gravitational-wave spectrum on the value of $g$ could also be said, with the necessary adaptations, about the parameters $m$ and $\lambda_{\chi}$. The dependence of the spectrum on the parameter $m$ is illustrated in Fig. 8. There, two spectra are shown, corresponding to the minimum and maximum values of $m$ allowed by the constraints (3) and (4) for fixed values of $\lambda_{\phi}, g$, and $\lambda_{\chi}$. For both values of $m$, the parameter

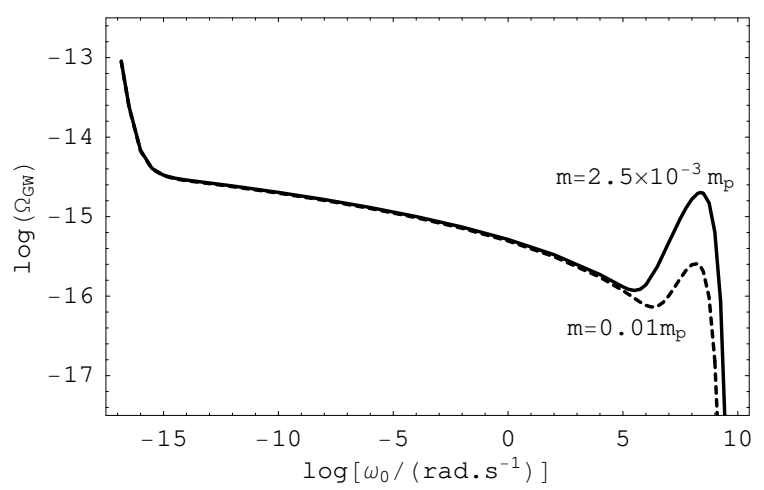

FIG. 8: Gravitational-wave spectrum for $\lambda_{\phi}=10^{-13}, g=$ $0.01, \lambda_{\chi}=1$, and $\mu=1$. The two curves correspond to the minimum and maximum values of $m$ allowed by the constraints (3) and (4), namely, $m=2.5 \times 10^{-3} m_{\mathrm{p}}$ and $m=0.01 m_{\mathrm{p}}$. In both cases $M$ is of the order of $10^{-14} m_{\mathrm{p}}$.

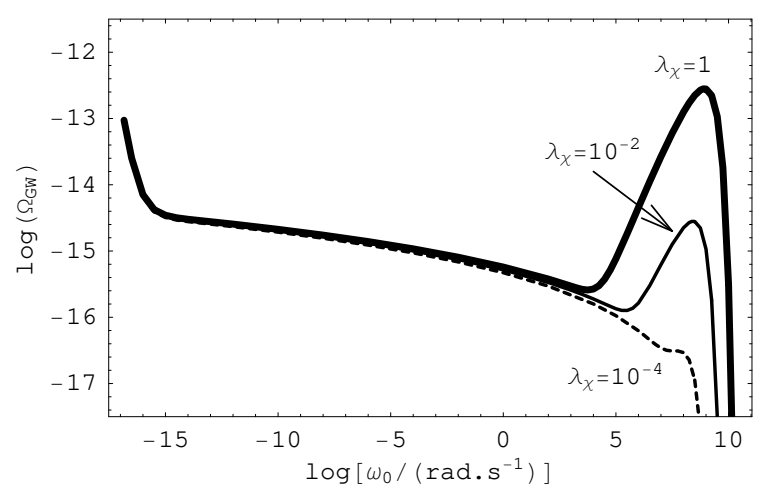

FIG. 9: Gravitational-wave spectrum for $\lambda_{\phi}=10^{-13}, g=$ $6.3 \times 10^{-4}, m=0.01 m_{\mathrm{p}}$, and $\mu=0.1$. The three curves correspond to $\lambda \chi=10^{-4}, 10^{-2}, 1$. The minimum value of $\lambda_{\chi}$, allowed by constraints (4) and (6), is $1.5 \times 10^{-5}$. In all cases $M$ is of the order of $10^{-14} m_{\mathrm{p}}$.

$\mu$ is chosen such that constraint (6) is satisfied. As we see in this figure, the height of the peak, located at high frequencies, increases as the parameter $m$ decreases. In Fig. 9, three gravitational-wave spectra are shown for different values of $\lambda_{\chi}$ and fixed values of $\lambda_{\phi}, g, m$ and $\mu$. As expected, the height of the peak increases as the parameter $\lambda_{\chi}$ increases. In all cases considered above, the parameter $M$ is of the order of $10^{-14} m_{\mathrm{p}}$.

Let us now turn to the analysis of the influence of the dissipation parameter $\mu$ on the duration of the kination period and, consequently, on the height of the high-frequency peak of the gravitational-wave spectrum. Remember that the parameter $\mu$ is bounded from below by the condition that the motion of $\phi$ is not affected by $\chi$ [see Eq. [6)]. We also require that this parameter is smaller than a critical value, $\mu_{\text {crit }}$, above which the oscillatory motion of the scalar field $\chi$ during the third stage of evolution would become over-damped. For values of the dissipation parameter $\mu$ lying in the interval 


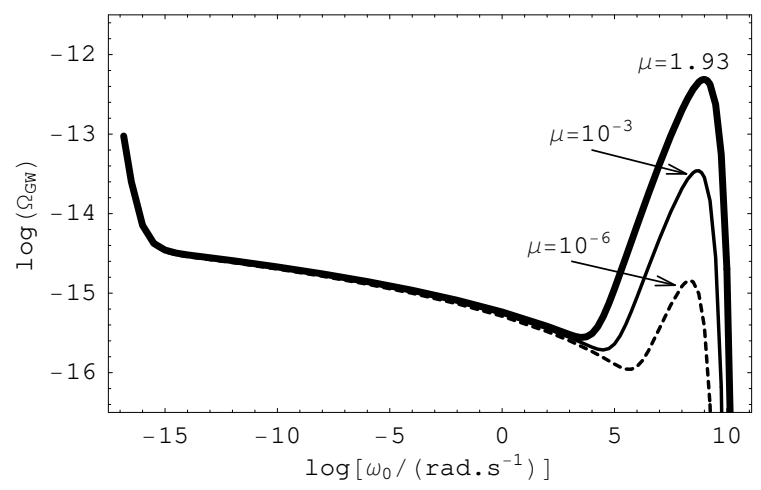

FIG. 10: Gravitational-wave spectrum for $\lambda_{\phi}=10^{-13}, g=$ $6.3 \times 10^{-4}, m=0.01 m_{\mathrm{p}}$, and $\lambda \chi=1$. The three curves correspond to $\mu=\mu_{\text {crit }}=1.93, \mu=10^{-3}$, and $\mu=10^{-6}$. Constraint (6) requires that $\mu \gg 10^{-7}$. In all cases $M$ is of the order of $10^{-14} m_{\mathrm{p}}$.

$0.2 \lambda_{\phi}^{1 / 6}\left(m_{\mathrm{p}} / m\right)^{2 / 3} g^{5 / 3} \lambda_{\chi}^{-1} \ll \mu<\mu_{\text {crit }}$, the duration of the kination period decreases as $\mu$ decreases. This is due to the fact that for smaller values of $\mu$ the energy transfer of the scalar field $\chi$ to the radiation fluid proceeds slower, with the consequence that the energy density of radiation begins to decrease as $a^{-4}$ later. Therefore, after the complete decay of the field $\chi$, the energy density of radiation is higher in the case of small $\mu$ and the time it takes for this energy density to dominate the dynamics of the Universe is shorter, implying that the kination period is also shorter. In Fig. 10 we plot three gravitational-wave spectra for different values of $\mu$, holding fixed the other parameters of the model. We see that the height of the peak in the high-frequency region of the spectrum increases as the dissipation parameter increases, achieving its maximum value for $\mu=\mu_{\text {crit }}$.

To complete our analysis of the influence of the various parameters of the potential $U(\phi, \chi)$ on the gravitationalwave spectrum, we point out that the parameter $\lambda_{\phi}$ determines the overall vertical displacement of the spectrum (see Fig. 11). Note that the value of $\lambda_{\phi}$ also affects the height of the peak, contrarily to the situation in original quintessential inflationary model, where only the lowand intermediate-frequency regions of the spectrum were pushed down (up) by an decrease (increase) of $\lambda_{\phi}$ (see Fig. 4 of Ref. [11]).

To finish this section, let us point out that, for the allowed values of the different parameters of the model, all gravitational-wave spectra satisfy, by far, the integral bound imposed by primordial nucleosynthesis [32, 37],

$$
\int_{\omega_{n}}^{\infty} \Omega_{\mathrm{GW}} d \ln \omega<1.1 \times 10^{-5},
$$

where $\omega_{n} \approx 10^{-9} \mathrm{rad} / \mathrm{s}$.

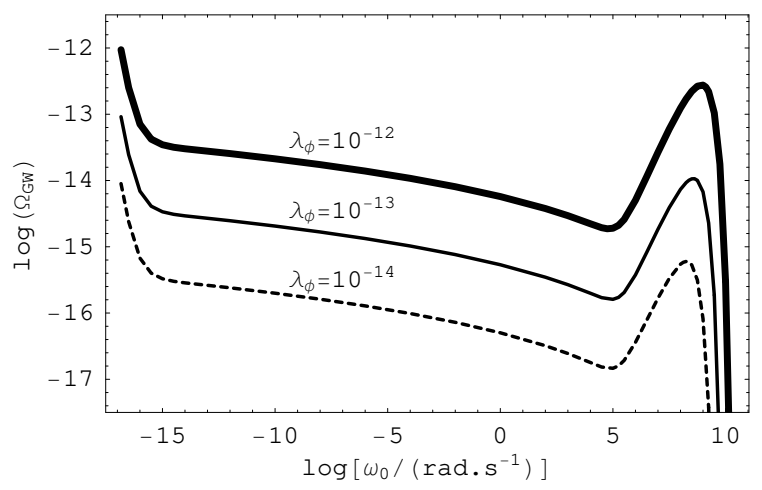

FIG. 11: Gravitational-wave spectrum for $g=0.002, m=$ $0.01 m_{\mathrm{p}}, \lambda \chi=1$, and $\mu=0.1$. The three curves correspond to $\lambda_{\phi}=10^{-14}, 10^{-13}, 10^{-12}$. In all cases $M$ is of the order of $10^{-14} m_{\mathrm{p}}$.

\section{CONCLUSIONS}

In this work we have investigated the generation of gravitational waves in the hybrid quintessential inflationary model. In this model, recently proposed by BasteroGil et al. [1], a unified description of inflation and dark energy is achieved with a single scalar field $\phi$ playing both roles of inflaton and quintessence, while reheating takes place in the usual way, through the complete decay of another scalar field, $\chi$, rather than by gravitational particle production, as in the original quintessential inflationary model of Peebles and Vilenkin [2].

The hybrid-like quintessential inflationary potential, $U(\phi, \chi)$, contains several parameters which are constrained by measurements of the cosmic microwave background radiation and dark energy, as well as by certain restrictions imposed on the behavior of the scalar field $\chi$, namely, that it responds quickly enough to changes in the potential $U(\phi, \chi)$ and that it does not influence significantly the evolution of the inflaton/quintessence field $\phi$.

Another relevant parameter of the model is the dissipation parameter which regulates the rate at which energy is transferred from the scalar field $\chi$ to radiation. This parameter is constrained from below by the condition that the motion of $\phi$ is not influenced significantly by $\chi$ and from above by the requirement that the oscillations of $\chi$ around the minimum of the potential are not over-damped.

For the allowed values of the above-mentioned parameters, we have calculated the full gravitational-wave energy spectrum using the method of continuous Bogoliubov coefficients. Such spectra carry, at high frequencies, a clear signature of quintessential inflation, namely, a sharp rise of the gravitational-wave spectral energy density parameter $\Omega_{\mathrm{GW}}$. This distinctive feature of the spectrum is due to the existence of a post-inflationary kination period, during which the kinetic energy of the inflaton field dominates the evolution of the Universe [10]. As we have shown in this paper, the duration of the ki- 
nation period depends crucially on the parameters of the hybrid-like potential, $g, m$ and $\lambda_{\chi}$, as well as on the dissipation parameter $\mu$. Namely, if $g$ and $m$ decrease $\left(\lambda_{\chi}\right.$ increases), reheating becomes less efficient, with the consequence that the kination period becomes longer. In this case, the peak in the high-frequency region of the gravitational-wave spectrum becomes more pronounced (see Figs. 6, 8 and 9). Similarly, if $\mu$ increases, the duration of the kination period also increases, implying a higher peak in the spectrum (see Fig. 10).

In the original quintessential inflationary model [2], the kination period is quite long, implying a pronounced peak in the high-frequency region of the gravitationalwave spectrum [11]. In order to avoid a conflict with the integral bound imposed by primordial nucleosynthesis [see Eq. (29)], in the original model one has to require that the number of scalar fields reheating the Universe is greater than about $10^{2}$ [2], meaning that the minimal Grand Unification Theory is not enough to accommodate quintessential inflation. This situation contrasts with the one occurring in the hybrid quintessential inflationary model. As we have shown in this paper, for the allowed values of the parameters of the model, the primordial-nucleosynthesis bound is satisfied, by far, by any gravitational-wave spectrum. Indeed, even when all parameters of the model are pushed to their extreme values, the gravitational-wave spectral energy density parameter $\Omega_{\mathrm{GW}}$ does not grow above $10^{-12}$. This important difference between the gravitational-wave spectrum of the original model and its hybrid variant is due to the fact that in the latter the duration of the kination period is much shorter, implying a smaller peak in the gravitational-wave spectrum.

The values of the different parameters also determine the frequency at which the gravitational-wave spectral energy density parameter $\Omega_{\mathrm{GW}}$ starts to rise at the highfrequency region of the spectrum. For extreme values of the parameters, this frequency can be as low as $10^{2} \mathrm{~Hz}$. Despite the fact that this frequency falls within the frequency range at which terrestrial laser-interferometer gravitational-wave detectors operate, a detection in a near future is improbable, due to the very low value of $\Omega_{\mathrm{GW}}$, about $10^{-15}-10^{-16}$.

In the gravitational-wave energy spectrum, the maximum of the peak is firmly located in the $\mathrm{MHz}-\mathrm{GHz}$ region and, within the hybrid quintessential inflationary model, can reach values of about $\Omega_{\mathrm{GW}} \simeq 10^{-12}$. A search for the primordial gravitational-wave background at this range of frequencies was initiated a few years ago, with both microwave-cavity detectors [38, 39] and interferometric detectors [40]. We deem the development of such detectors to be of the utmost importance, since they will open a direct window to the very early Universe, allowing us to test our theoretical ideas about the inflationary and post-inflationary epochs.

\section{Acknowledgments}

The authors thank R. Potting for interesting discussions. This work was supported in part by the Fundação para a Ciência e a Tecnologia, Portugal.
[1] M. Bastero-Gil, A. Berera, B. M. Jackson, and A. Taylor, Phys. Lett. B 678, 157 (2009).

[2] P. J. E. Peebles and A. Vilenkin, Phys. Rev. D 59, 063505 (1999).

[3] B. Spokoiny, Phys. Lett. B 315, 40 (1993).

[4] M. Joyce, Phys. Rev. D 55, 1875 (1997).

[5] L. H. Ford, Phys. Rev. D 35, 2955 (1987).

[6] G. Felder, L. Kofman, and A. Linde, Phys. Rev. D 60, 103505 (1999).

[7] A. Linde, Phys. Rev. D 49, 748 (1994).

[8] L. P. Grishchuk, Ann. Acad. Sci. 302, 439 (1977).

[9] B. L. Hu and L. Parker, Phys. Lett. A 63, 217 (1977).

[10] M. Giovannini, Phys. Rev. D 58, 083504 (1998).

[11] M. Giovannini, Phys. Rev. D 60, 123511 (1999).

[12] A. Riazuelo and J.-P. Uzan, Phys. Rev. D 62, 083506 (2000).

[13] V. Sahni, M. Sami, and T. Souradeep, Phys. Rev. D 65, 023518 (2001).

[14] H. Tashiro, T. Chiba, and M. Sasaki, Class. Quantum Gravity 21, 1761 (2004).

[15] L. Parker, Phys. Rev. 183, 1057 (1969).

[16] A. B. Henriques, Phys. Rev. D 49, 1771 (1994).

[17] R. G. Moorhouse, A. B. Henriques, and L. E. Mendes, Phys. Rev. D 50, 2600 (1994).

[18] L. E. Mendes, A. B. Henriques, and R. G. Moorhouse, Phys. Rev. D 52, 2083 (1995).
[19] A. B. Henriques, Class. Quantum Gravity 21, 3057 (2004); 24, 6431(E) (2007).

[20] P. M. Sá and A. B. Henriques, Phys. Rev. D 77, 064002 (2008).

[21] P. M. Sá and A. B. Henriques, Gen. Relativ. Gravit. 41, 2345 (2009).

[22] A. B. Henriques, R. Potting, and P. M. Sá, Phys. Rev. D 79, 103522 (2009).

[23] T. L. Smith, M. Kamionkowski, and A. Cooray, Phys. Rev. D 73, 023504 (2006).

[24] E. Komatsu et al., "Seven-Year Wilkinson Microwave Anisotropy Probe (WMAP) Observations: Cosmological Interpretation, arXiv:1001.4538 2 [astro-ph.CO].

[25] J. Yokoyama and K. Maeda, Phys. Lett. B 207, 31 (1988).

[26] L. P. Grishchuk, Sov. Phys. JETP 40, 409 (1974).

[27] A. A. Starobinskii, JETP Lett. 30, 682 (1979).

[28] L. F. Abbott and D. D. Harari, Nucl. Phys. B 264, 487 (1986).

[29] B. Allen, Phys. Rev. D 37, 2078 (1988).

[30] V. Sahni, Phys. Rev. D 42, 453 (1990).

[31] L. P. Grishchuk and M. Solokhin, Phys. Rev. D 43, 2566 (1991).

[32] B. Allen, in Proceedings of the Les Houches School on Astrophysical Sources of Gravitational Waves (Les Houches, France, 1995), edited by J.-A. Marck and J.-P. La- 
sota (Cambridge University Press, Cambridge, England, 1997), p. 373.

[33] S. Kuroyanagi, T. Chiba, and N. Sugiyama, Phys. Rev. D 79, 103501 (2009).

[34] F. A. Jenet et al., Astrophys. J. 653, 1571 (2006).

[35] J. W. Armstrong, L. Iess, P. Tortora, and B. Bertotti, Astrophys. J. 599, 806 (2003).

[36] B. P. Abbott et al. (LIGO and Virgo Collaborations),
Nature 460, 990 (2009).

[37] M. Maggiore, Phys. Rep. 331, 283 (2000).

[38] Ph. Bernard, G. Gemme, R. Parodi, and E. Picasso, Rev. Sci. Instrum. 72, 2428 (2001).

[39] A. M. Cruise and R. M. J. Ingley, Class. Quantum Gravity 23, 6185 (2006).

[40] T. Akutsu et al., Phys. Rev. Lett. 101, 101101 (2008). 